\title{
Updates to the Oxford Space Environment Goniometer to Measure Visible Wavelength Bidirectional Reflectance Distribution Functions in Ambient Conditions
}

\author{
R. J. Curtis, T. J. Warren, N. E. Bowles ${ }^{1}$. \\ ${ }^{1}$ Atmospheric, Oceanic and Planetary Physics, Department of Physics, University of Oxford, Oxford, \\ United Kingdom, OXI 4BH.
}

Corresponding author: R.J.Curtis_rowan.curtis@physics.ox.ac.uk; neil.bowles@physics.ox.ac.uk; tristram.warren@physics.ox.ac.uk.

Understanding how the surfaces of airless planetary bodies—-such as the Moon — scatter visible light enables constraints to be placed on their surface properties, and top boundary layer inputs to be set within thermal models. Remote sensing instruments-such as Diviner onboard the Lunar

Reconnaissance Orbiter-measure thermal emission and visible light scattering functions across visible $(\sim 0.38-0.7 \mu \mathrm{m})$ to thermal infrared (TIR) wavelengths $(\sim 0.7-350 \mu \mathrm{m})$. To provide ground support measurements for such instruments, the Oxford Space Environment Goniometer (OSEG) was built. Initially, OSEG focused on measuring TIR directional emissivity functions for regolith and regolith simulant samples in a simulated space environment, but it has recently been modified to measure visible wavelength Bidirectional Reflectance Distribution Functions (BRDFs) of samples in ambient conditions. Laboratory-measured BRDFs can be used 1) to test, and to help interpret models - such as the Hapke photometric model — and 2) as visible scattering function inputs for thermal models. This paper describes the modifications to, and initial calibration measurements taken by the Visible Oxford Space Environment Goniometer (VOSEG), with a 532nm laser, and details how this setup can be used to measure BRDFs of regolith and regolith simulant samples of airless planetary bodies.

\section{Introduction and Motivation}

Measuring Bidirectional Reflectance Distribution Functions (BRDFs) for planetary regolith and regolith simulant samples is useful in planetary science for the following reasons:

1) Testing and Interpreting the Hapke Model: The Hapke model is a commonly used photometric model in planetary reflectance data analysis because it is based on solutions to the radiative transfer equation, and because it has nine free parameters which relate to the composition and surface properties (such as porosity and surface roughness) of a particulate surface ${ }^{1-5}$. Visible reflectance/albedo measurements taken by remote sensing instruments such as the Diviner Radiometer (onboard the Lunar

Reconnaissance Orbiter (LRO)) can therefore be interpreted by comparing them with Hapke modelled BRDFs ${ }^{6,7}$. This provides a method by which compositional/physical property variations can be determined across varying regions of an airless bodies' surface ${ }^{6}$. Any deductions made using this method rely on the physicality of the free parameters of the model. These parameters have been updated over time, as further remote sensing and laboratory BRDF measurements have become available ${ }^{3,8-10}$. Hence, BRDF measurements for novel samples with known compositional and physical properties (i.e. knowledge of volume-average single scattering albedo- $w$; forward/backscattering parameters $-b$ and $c$; and porosity and surface roughness ${ }^{2,6}$ ) may help to further test-and improve the physicality of - the terms within the model ${ }^{11}$.

2) As inputs for Thermal Models: Laboratory BRDF measurements of regolith samplessuch as the Apollo lunar regolith samples returned from the lunar surface ${ }^{12}$ - provide more accurate scattering function inputs for thermal models than when using estimated functions, such as Lambertian ones ${ }^{11}$. The top layer temperature boundary conditions within thermal models are set by inputting BRDFs for the surface ${ }^{13}$. If these are inaccurate, it may lead to temperature discrepancies between modelled and remotely sensed temperatures, in particular for Permanently Shadowed Regions 
(PSRs), such as those in high-latitude $\left(>50^{\circ}\right)$ areas of the Moon ${ }^{14-16}$.

Points 1 and 2 motivate the modifications made to the OSEG instrument - which was initially built to measure Thermal Infrared (TIR) emission phase functions - so that it can measure visible wavelength BRDFs over a wide range of viewing angles $\left(0-70^{\circ}\right.$ reflectance angle, 0-60 incidence angle and 0$360^{\circ}$ azimuthal angle). How the requirements of the VOSEG instrument are derived from these two motivations is described in parts A, and $\mathrm{B}$.

\section{A. Experimental Requirements Derived from the Hapke Model \\ i. Introduction to the Hapke Model}

The Hapke photometric model predicts BRDFs for particulate surfaces, which describe how visible to near-infrared radiation is scattered over all viewing angles (incidence, reflectance, azimuthal ${ }^{1,2}$. The BRDF, given in Equation 1, is defined as the ratio of scattered radiation at the detector, compared to the collimated incident radiation which illuminates the surface, per unit area perpendicular to the direction of incidence ${ }^{2}$.

$$
\operatorname{BRDF}\left(\theta_{r}, \theta_{i}, \theta_{a}\right)=\frac{r\left(\theta_{r}, \theta_{i}, \theta_{a}\right)}{\cos \left(\theta_{i}\right)}
$$

Where $r\left(\theta_{r}, \theta_{i}, \theta_{a}\right)$ is reflectance ${ }^{2}$, given in Equation 2, and $\theta_{r}, \theta_{i}$ and $\theta_{a}$ are reflectance, incidence and azimuthal angles respectively (see Figure 1) ${ }^{2,17}$.

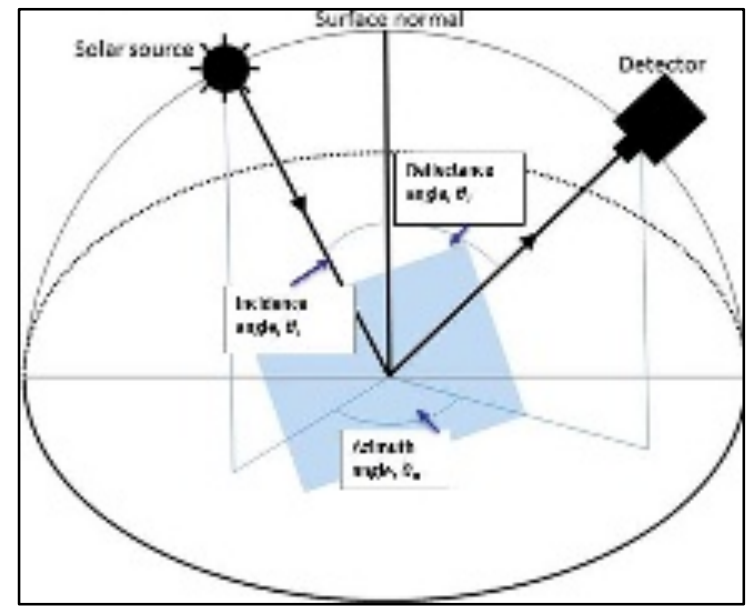

Figure 1: Definition of scattering angles for the Bidirectional Reflectance Distribution Function (BRDF) (Reproduced from Warren et al. (2017), Rev. Sci. Instrum., 88, 124502, with the permission of AIP publishing).

$$
\begin{aligned}
& r\left(\theta_{r}, \theta_{i}, \theta_{a}\right)=K \frac{w}{4 \pi} \frac{\mu_{r}}{\mu_{0}+1} p\left(\theta_{p}\right)[1+ \\
& \left.\left.B_{S 0} B_{S}\left(\theta_{p}\right)\right]+\left[H\left(\mu_{0} / K\right) H\left(\mu_{r} / K\right)-1\right]\right\}[1+ \\
& \left.B_{C 0} B_{C}\left(\theta_{p}\right)\right]
\end{aligned}
$$

Where, $K$ is porosity coefficient, $w$ is volumeaverage single scattering albedo, $\frac{\mu_{r}}{\mu_{0}}=\frac{\cos \left(\theta_{r}\right)}{\cos \left(\theta_{i}\right)}$, $p\left(\theta_{p}\right)$ is volume-average single-particle scattering function, $\theta_{p}$ is phase angle, $B_{S}$ is the Shadow-Hiding Opposition Effect (SHOE) part of the total opposition effect. $B_{S 0}$ is the amplitude of the SHOE effect, $B_{C 0}$ is the amplitude of the Coherent Backscattering Opposition Effect (CBOE) - the second part of the total opposition effect - and $H(x)$ is an approximation of the AmbartsumianChandrasekhar function as described in $\mathrm{Hapke}^{2}$. It is helpful to clarify what some of these parameters mean physically.

$\omega$ : average particle single scattering albedo, which quantifies the efficiency of an average regolith grain in scattering and absorbing light at a specific wavelength (Equation 3$)^{18}$.

$$
\omega=\frac{\sigma_{s c a}}{\sigma_{s c a}+\sigma_{a b s}}
$$

Where $\sigma_{s c a}$ and $\sigma_{a b s}$ are the scattering and absorption coefficients, respectively. They quantify the fraction of light scattered or absorbed by a medium. Hence, $0 \leq \omega \leq 1^{18}$.

$K$ : porosity coefficient, a measure of how porosity affects transmission within a medium. For particle sizes $>\lambda$ it is related to the filling factor, $\phi$ by Equation $4^{18}$.

$$
K=\frac{-\ln \left(1-\left(1.209 \phi^{\frac{2}{3}}\right)\right)}{\left(1.209 \phi^{\frac{2}{3}}\right)}
$$

$p\left(\theta_{p}\right)$ : the volume-average single-particle phase function, modelled as a HenyeyGreenstein (HG) function of one, two or three terms. It can also be modelled as a Legendre polynomial, but $\mathrm{HG}$ is used here as it describes complex scattering with equal accuracy to the Legendre polynomial, but with fewer parameters ${ }^{19}$. The three-term HG function is defined in Hapke ${ }^{2}$ and contains the forward/backscattering parameters $b$ and $c$. 


\section{ii. Experimental Validation of the Model and Previous BRDF Measurements}

In addition to modelling BRDFs for particulate planetary surfaces (i.e. to be used in thermal models), the Hapke model has also been used as a tool for purposes such as asteroid image corrections $^{4,5}$ and spectral unmixing ${ }^{20,21}$. For the latter two uses, the Hapke model has been validated through extensive laboratory studies $^{22}$, but for the former, only limited studies have been performed, such as those by Shepard and Helfenstein, in $2007^{22}$, and Foote et al., in $2010^{11}$.

Since the Hapke model was first described, various goniometer systems have measured BRDFs for a range of particulate samples such as regolith simulants ${ }^{23}$, regolith/ice mixtures ${ }^{24}$, terrestrial analogue materials and meteorites ${ }^{25}$. These include studies made using the Physikalisches Institut Radiometer Experiments (PHIRE-1 and PHIRE-2) ${ }^{23}$, the SHADOWS spectro-gonio radiometer ${ }^{25}$, and the Blacklab goniometer system ${ }^{26}$. In 2010, Foote et al. measured BRDFs of Apollo 11 (maria) and 16 (highlands) regolith samples using the Bloomsburg University Goniometer $(\mathrm{BUG})^{11,27}$. As opposed to previous studies of Apollo samples, which only measured a very limited range viewing angles (i.e. with a fixed reflectance angle or incidence angle ${ }^{28}$, these measurements were made over a wide range of viewing angles $\left(0-75^{\circ}\right.$ incidence angle, $0-80^{\circ}$ reflectance angle and $0-360^{\circ}$ azimuthal) $)^{11,12}$. The study concludes by suggesting that further BRDF measurements of Apollo regolith samples are required to deconvolve the influence of porosity on the measured BRDFs of the samples. Within their publications Hapke $^{2}$, Shepard and Helfenstein ${ }^{22}$ and Foote et al. ${ }^{11}$ all suggest more goniometry measurements are required to further test the Hapke model, and to further understand how physical properties such as porosity and surface roughness influence visible light scattering for planetary regolith and simulant samples $^{11}$.

\section{iii. Measuring the Opposition Effect}

The Hapke BRDF model includes the opposition effect, a rapid nonlinear brightness increase when phase angle nears $\theta_{p}=0^{\circ}{ }^{18}$.

The total opposition effect is an amalgamation of the Coherent Backscattering Opposition Effect (CBOE) and the Shadow-Hiding Opposition Effect (SHOE) ${ }^{29}$. It was first noted in the autobiography of the artist Benvenuto Cellini in the sixteenth century ${ }^{30}$, and can be observed in images taken during the Apollo missions, with bright haloes appearing around the shadows cast by the astronauts' heads (Figure 2) $)^{18}$.

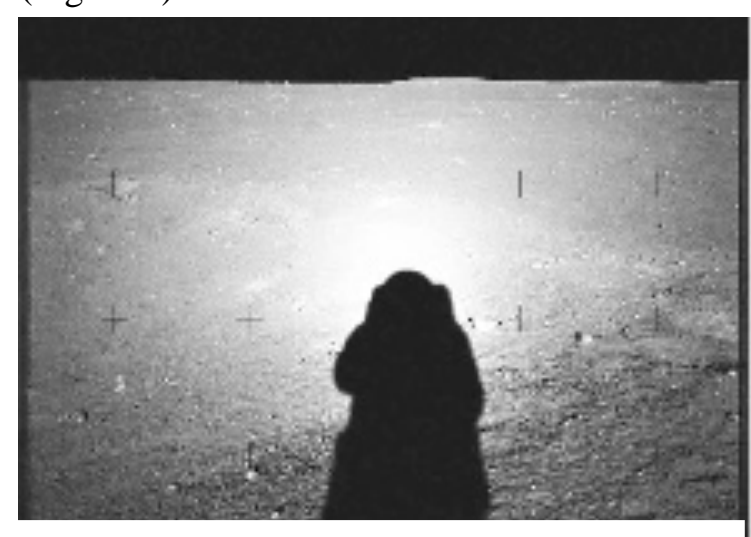

Figure 2: This image (AS-11-40-5882) demonstrates the Opposition Effect: At close to zero phase angle, a bright halo is observed around the shadow of an Apollo 11 astronaut's head (image credit-NASA).

The opposition effect can be measured in the laboratory, but to be able to do so, a goniometer must measure phase angles $<\sim 10^{\circ}$, as demonstrated in Figure 3, which shows how the $\mathrm{SHOE}$ variable, $B_{S}$ varies within the model, as it approaches $\theta_{p}=0^{\circ} 31$.

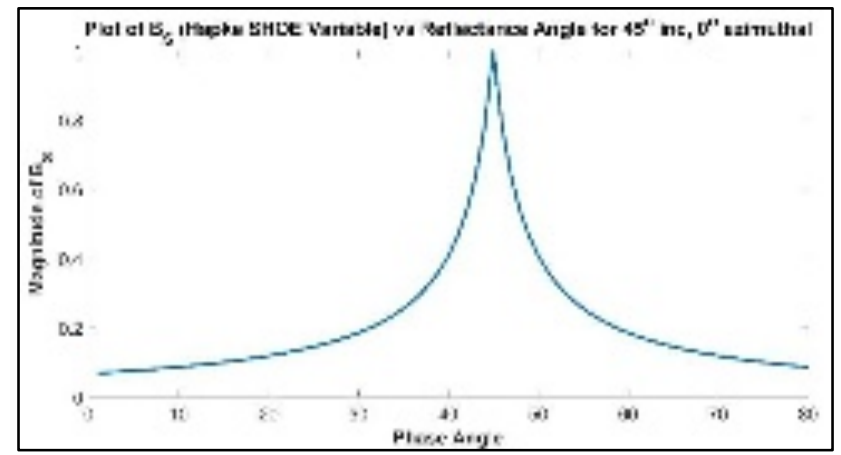

Figure 3: $B_{S}$, the Shadow-Hiding Opposition Effect variable within the Hapke model vs reflectance angle, for $45^{\circ}$ incidence angle; $0^{\circ}$ azimuthal angle; $w=0.2883$;

Henyey-Greenstein coefficients, $b=0.2917, c=0.4052$ and $h s=0.03$ as given for the Apollo 16 (68810) sample in the Foote et al study. 
Therefore, to test the opposition effect terms within the Hapke model, the first requirement for a goniometer system is to be able to measure as low phase angles $\left(<10^{\circ}\right)$ as possible.

\section{iv. Measuring at High Phase Angles}

Foote et al. showed that for Apollo regolith samples, the Hapke model predicts the most prominent deviations - between laboratory measurements and models for Lambert Albedo (the ratio of the measured reflectance relative to that of a Lambertian surface, related to the BRDF by Equation 5) - at reflectance angles $>60^{\circ}$ (Figure 4$)^{11,32}$. Foote et al. suggest these deviations are due to porosity variations for the measured samples ${ }^{11}$. Hence, to study how porosity variations affect measured BRDFs, a goniometer must measure reflectance angles $>60^{\circ}$.

$$
A_{L}\left(\theta_{r}, \theta_{i}, \theta_{a}\right)=\pi \frac{B R D F\left(\theta_{r}, \theta_{i}, \theta_{a}\right)}{\cos \left(\theta_{i}\right)}
$$

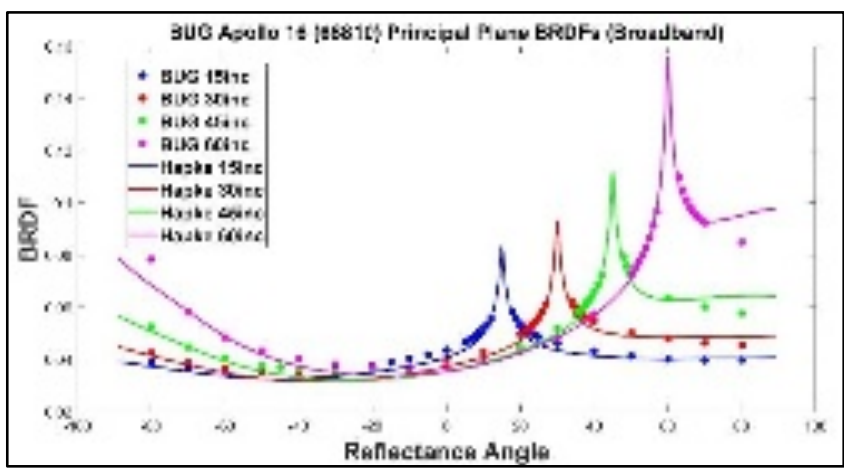

Figure 4: Apollo 16 (68810) BRDF plots for laboratory data (dots) and Hapke modelled plots (solid lines) measured by BUG (in the Foote et al, 2010 study). For the Hapke model, single scattering albedo, $w=0.2883$, and Henyey-Greenstein coefficients, $b=0.2917$ and $c=0.4052$.

In addition to porosity, the influence of surface roughness is included within the model by multiplying $r\left(\theta_{r}, \theta_{i}, \theta_{a}\right)$ by a shadowing factor, $S\left(\theta_{r}, \theta_{i}, \theta_{p}\right)$, given by Equation 6 , which is dependent on viewing angle, and the slope angle of the surface, $\bar{\theta}^{10}$ :

$$
\begin{aligned}
& S\left(\theta_{r}, \theta_{i}, \theta_{a}\right) \\
& =\frac{\mu_{r}}{\eta_{r}\left(\theta_{r}\right)} \frac{\mu_{0}}{\eta_{0 r}\left(\theta_{i}\right)} \frac{\chi(\bar{\theta})}{1-f\left(\theta_{p}\right)+f\left(\theta_{p}\right) \chi(\bar{\theta})\left[\frac{\mu_{0}}{\eta_{0 r}\left(\theta_{i}\right)}\right]}
\end{aligned}
$$

Where $f\left(\theta_{p}\right)=\exp \left(-2 \tan \left(\frac{\theta_{p}}{2}\right)\right), \chi(\bar{\theta})=$ $\frac{1}{\left(1+\pi \tan ^{2}(\bar{\theta})\right)^{1 / 2}}$, and $\eta_{r}\left(\theta_{r}\right)$ and $\eta_{0 r}\left(\theta_{i}\right)$ are the effective cosines which appear within the shadowing factor - for reflectance angle and incidence angle, respectively - as defined in Hapke $^{2}$.

Reflectance (with surface roughness considerations included) is thus given by Equation 7:

$$
r_{R}\left(\theta_{r}, \theta_{i}, \theta_{a}\right)=r\left(\theta_{r}, \theta_{i}, \theta_{a}\right) S\left(\theta_{r}, \theta_{i}, \theta_{a}\right)
$$

Variations due to surface roughness are most prominent for high reflectance angles $\left(>60^{\circ}\right)$, as demonstrated in Figure $5^{2}$.

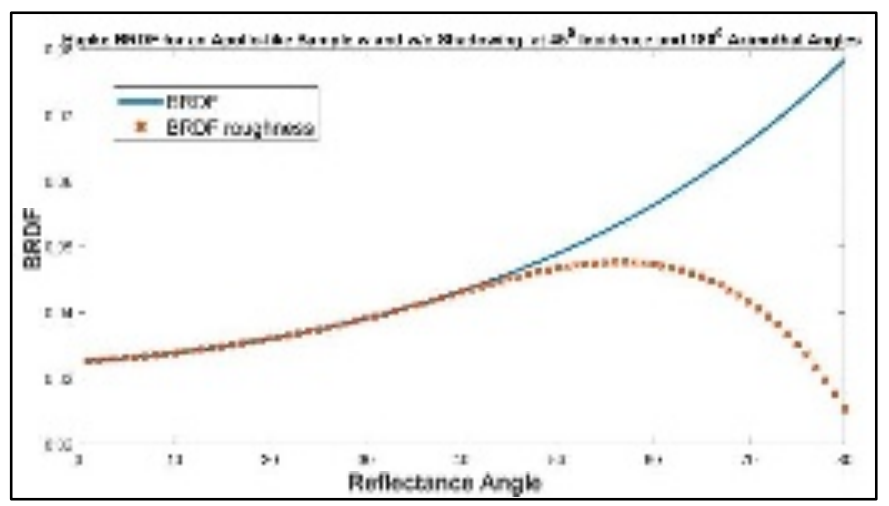

Figure 5: Hapke Modelled BRDFs for an Apollo-like regolith sample, with shadowing (orange crosses), and without shadowing (solid line), for $45^{\circ}$ incidence and $180^{\circ}$ azimuthal angles. Slope angle $=20^{\circ}$, particle size $=70 \mu \mathrm{m}$, filling factor $=0.5$, single scattering albedo, $w=0.2883$, and HenyeyGreenstein coefficients, $b=0.2917$ and $c=0.4052$.

Therefore-1) to corroborate Foote et al.'s measured BRDFs and 2) to test the Hapke model (with and without the surface roughness shadowing function), VOSEG requires the capability to measure low phase $\left(<10^{\circ}\right)$ and high reflectance angles $\left(>60^{\circ}\right)$. Measurements at these high and low phase angles become particularly important if studies are to be done on the opposition effect, or on how porosity and surface roughness variations influence BRDFs.

\section{B. Experimental Requirements Derived from Thermal Models}

By comparing 3D Thermal Model (3DTM) surface temperatures of planetary bodies, to those measured by remote sensing instruments (e.g. the Diviner Lunar Radiometer and the 
OSIRIS-REx Thermal Emission Spectrometer (OTES)) it is possible to constrain surface composition, surface roughness, emissivity, density/porosity, albedo, and subsurface (up to $\sim 0.5 \mathrm{~m}$ deep) temperatures ${ }^{33-36}$.

Key inputs for modelling surface/subsurface temperature profiles for airless bodies include density profiles, thermal conductivity functions and TIR/visible wavelength phase functions for specific regolith/rock-regolith mixtures ${ }^{37}$. For lunar thermal models, density and thermal conductivity boundary conditions are typically set using studies of Apollo core samples $^{38}$. (Although recent work has suggested thermal conductivity profiles for regolith samples at $<100 \mathrm{~K}$ need to be updated $^{39}$.) But for TIR emission phase functions and BRDFs for the lunar surface only limited laboratory measurements, such as those done by Foote et al., have previously been made ${ }^{3,11,40}$.

In high-latitude $\left(>60^{\circ}\right)$ regions of the Moon water ice may exist in permanently shadowed regions (PSRs) ${ }^{37,41}$, which occur due to the Moon's low ecliptic obliquity $\left(\sim 1.53^{\circ}\right){ }^{42}$. In such PSR regions, surface temperature is dominantly influenced by thermal re-emission and visible light scattering from illuminated regions (see Figure 6) ${ }^{17,41}$.

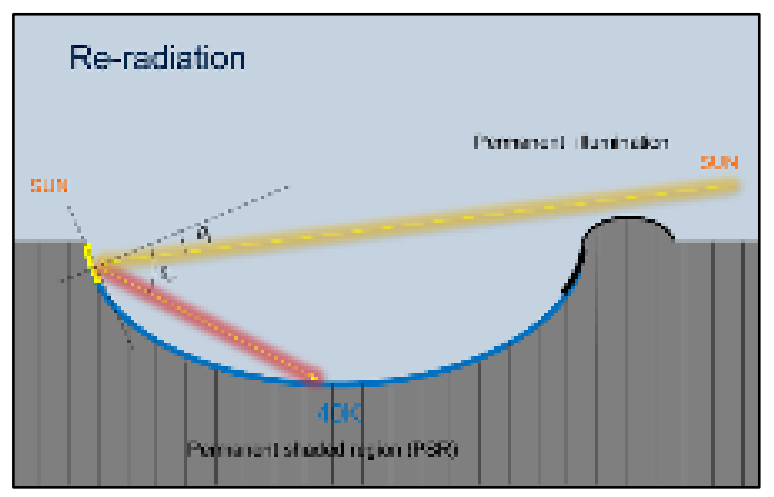

Figure 6: A 2-Dimensional slice of a gridded cratered region of the Moon, showing how light scattering from illuminated regolith is reflected into permanently shadowed regions of the crater (Reproduced from Warren et al. (2017), Rev. Sci. Instrum., 88, 124502, with the permission of AIP publishing).

Remote sensing temperature measurements taken by the Diviner Lunar Radiometer show temperature discrepancies of up to $\sim 15 \mathrm{~K}$ within and around PSRs, when compared with
$3 \mathrm{DTMs}^{16,37}$. Using laboratory-measured BRDFs and TIR emission phase functions within thermal models - instead of assuming them to be Lambertian or estimating them numerically - may reduce these temperature discrepancies ${ }^{11,17}$. To capture how reflectance and reemission affects PSRs for as large a range of crater reflectance angles as possible, such BRDF and TIR emission phase functions of Apollo regolith and lunar regolith simulant samples must be measured for as wide a range of viewing angles as possible. Provided phase angles $<10^{\circ}$ and reflectance angles $>60^{\circ}$ are measurable, the remaining parts of the BRDF curves can be extrapolated using the Hapke model $^{11}$.

\section{Instrumentation. \\ A. The Visible Oxford Space Environment Goniometer}

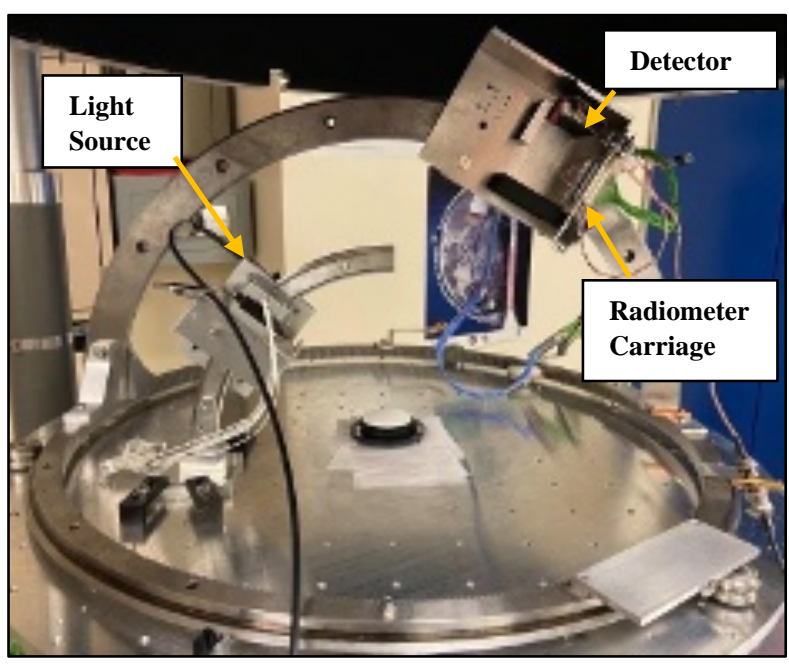

Figure 7: The Oxford Space Environment Goniometer measuring White Spectralon in visible wavelength mode.

Figure 7 shows the basic parts of the Visible Oxford Space Environment Goniometer (VOSEG). The original version of the instrument, OSEG, was designed to make automated measurements at TIR wavelengths, but the system is now capable of measuring across visible to far-infrared wavelengths ( $\sim 38-350 \mu \mathrm{m})$, over a range of viewing angles $\left(0-60^{\circ}\right.$ incidence angle, $0-70^{\circ}$ reflectance angle, $0-360^{\circ}$ azimuthal angle, $\geq 4^{\circ}$ phase angle $)^{43}$.

OSEG, for TIR, is surrounded by a cold shield and is contained within a vacuum chamber, 
allowing measurements to be made in simulated lunar environment conditions (i.e. under $10^{-6} \mathrm{mbar}$ vacuum and at $<150 \mathrm{~K}$ temperatures $)^{43}$. Visible wavelength BRDF measurements, however, do not require vacuum conditions (although vacuum measurements are possible), or to be made at lunar-like temperatures, and so are taken in ambient conditions, as this is more time efficient and the thermal background does not significantly contribute to visible BRDF measurements ${ }^{22}$. Typically, one measurement run (of three half-hemisphere measurements which can then be averaged) takes $\sim 30$ minutes. For BRDF measurements, automated angle variations are achieved as follows:

- Azimuthal angle variation achieved by rotating a $650 \mathrm{~mm}$ diameter steel ring around the sample using motors.

- Reflectance angle variation achieved by moving the radiometer carriage along a half-hemisphere $\left(180^{\circ}\right)$ steel ring segment using motors. The distance between the target and the detector is $\sim 310 \mathrm{~mm}$ (and $\sim 245 \mathrm{~mm}$ to the end of the baffle).

- Incidence angle variation is achieved by moving the quarter-hemisphere $\left(90^{\circ}\right)$ steel ring segment to which the fiber optic holder plate is screwed. $0-45^{\circ}$ can be achieved with fiber holder 1 (clear). For higher incidence angles $\left(>45^{\circ}\right)$, fiber holder 2 (red) must be used, due to spatial constraints. The distance between the target and the fiber optic output is $\sim 184 \mathrm{~mm}$ (and $\sim 158 \mathrm{~mm}$ to the end of the baffle).

The output of the photodiode is passed into a lock-in amplifier (SR830 - Stanford Research Systems) to demodulate the output from the chopper reference signal. Chopping the light (at $130 \mathrm{~Hz}$ ) and using the lock-in amplifier (referenced to $130 \mathrm{~Hz}$ ) removes the signal due to background light. The output is then recorded using a MATLAB control code on a PC. The specifications of VOSEG's possible viewing angles are listed in Table I.

\begin{tabular}{|l|l|l|}
\hline Angle & $\begin{array}{l}\text { Minimum } \\
\text { Requirement }\end{array}$ & $\begin{array}{l}\text { Possible } \\
\text { with } \\
\text { VOSEG }\end{array}$ \\
\hline Azimuthal & $0-360^{\circ}$ & $0-360^{\circ}$ \\
\hline Reflectance & $0-60^{\circ}$ & $0-70^{\circ}$ \\
\hline Incidence & $0-60^{\circ}$ & $0-60^{\circ}$ \\
\hline Phase & $5-120^{\circ}$ & $\sim 4-130^{\circ}$ \\
\hline
\end{tabular}

Table I: Angular capabilities of the Visible Oxford Space Environment Goniometer.

Phase angles down to $\sim 4^{\circ}$, and reflectance angles up to $70^{\circ}$ can be measured using this setup, as shown in Figure 8.

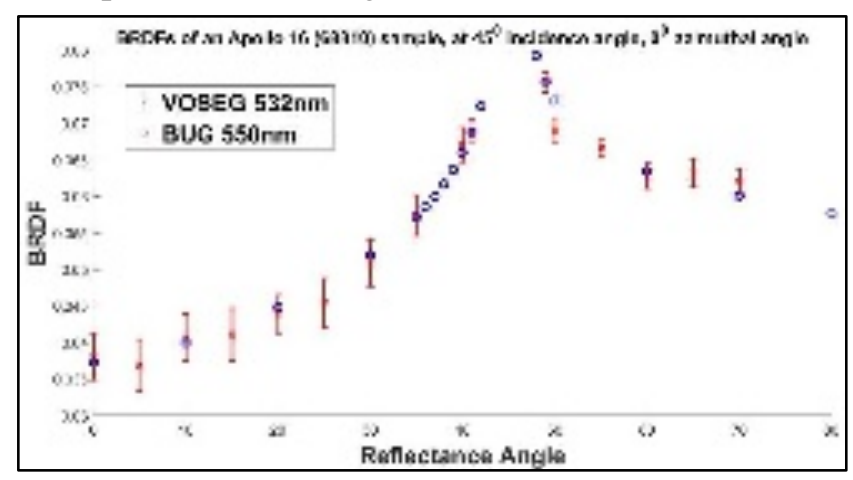

Figure 8: BRDF measurements made by VOSEG of an Apollo 16 (68810) regolith sample, at $45^{\circ}$ incidence angle, $O^{\circ}$ azimuthal angle, down to $4^{\circ}$ phase angle and up to $70^{\circ}$ reflectance angle, and compared to BUG. (VOSEG data in red, BUG data in blue.)

Measuring angles $<4^{\circ}$ is not possible with the VOSEG setup - despite it being as 'slimline' as possible — due to the light source obscuring the detector. Measurements of phase angles $<4^{\circ}$ are experimentally possible, but require a beam splitter, or a larger goniometer ${ }^{44}$.

\section{B. Radiometer Carriage and Detector Field of View}

VOSEG's radiometer carriage contains a detector holder plate which screws onto the main carriage. This holds the visible wavelength photodiode (RS Components BPW21R) in place, which looks straight down at the sample. An additional $15 \times 60 \times 75 \mathrm{~mm}$ Aluminum baffle plate, with a $6.2 \mathrm{~mm}$ diameter hole is then screwed to the lower part of the carriage, to restrict the Field of View (FOV) of the detector. Black card is then mounted 
around the radiometer carriage to reduce the stray light incident on the photodiode. Figure 9 shows an image of the setup.

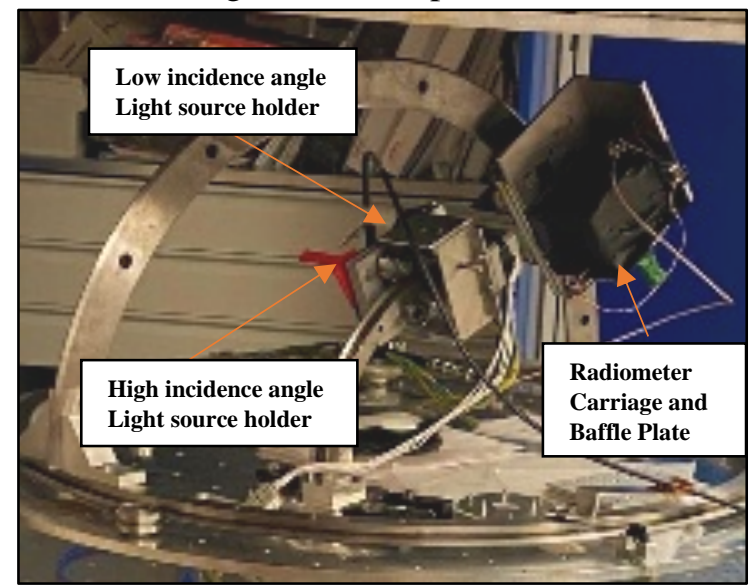

Figure 9: Image of VOSEG taking measurements of White Spectralon. Black card surrounds the radiometer carriage to reduce stray light and both light source holders can be seen.

A 2D slice of the circular FOV of the detectorbaffle setup is shown in Figure 10, with dotted lines showing one standard deviation from the centre. The effective FOV is $\sim 20 \mathrm{~mm}$ and this was measured by moving a pinhole of lightcreated by mounting a baffle with a $1 \mathrm{~mm}$ outer diameter hole in front of the laser-across the detector FOV in the $\mathrm{x}$ - and y-planes . (NB: An ideal FOV would be as flat as possible-i.e. a 'top hat'.)

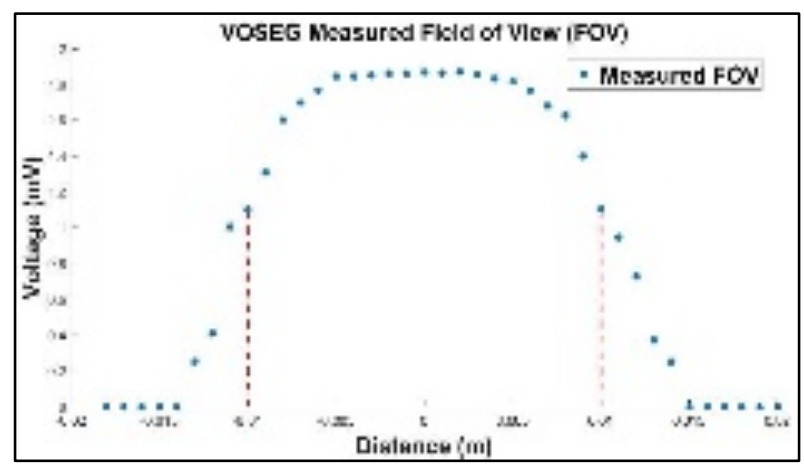

Figure 10: A 2D slice of the Oxford Space Environment Goniometer's measured field of view for the visible wavelength setup. Blue datapoints are the measured voltages in millivolts and the red dashed line indicates the effective Field of View (one standard deviation from the centre).

\section{Light Source and Illuminated Spot}

Currently, VOSEG can take BRDF measurements with two light sources: 1) a $532 \mathrm{~nm}$ ThorLabs CPS532 ${ }^{45}$ laser-which is elliptically polarized with a Polarization
Extinction Ratio of $4 \mathrm{~dB}$, but this did not lead to variations $\geq$ the standard error in measured BRDF for White Spectralon ${ }^{46}$-or 2) a stabilized Bentham IL1 broadband Quartz Tungsten Halogen (QTH) lamp ${ }^{47}$. The incident light is chopped at $130 \mathrm{~Hz}$ and launched down a $2 \mathrm{~m}$ single mode fiber optic cable (Edmund Optics \#36-365). This fiber is fixed in place using a 3D printed fiber optic cable holder, which screws onto the incidence angle quarterhemisphere steel ring. The fiber optic is then fed into a three-stage 3D printed baffle structure, with a metal baffle plate attached at the end of it, to restrict the illuminated spot size to $\sim 9 \mathrm{~mm}$ (outer diameter), when the fiber is at $0^{\circ}$ incidence (looking straight down). To ensure the full illumination spot is within the FOV at all incidence angles $\left(0-60^{\circ}\right)$, the effective illumination spot must be mapped, and kept to a size of $10 \%$ less than the detector's FOV. This is because the illumination spot is aligned to be within the centre of the FOV for each experimental run, and there is an uncertainty in this alignment of $\pm 1 \mathrm{~mm}$, which corresponds to $\pm 5 \%$ of the detector's effective FOV (see Figure 10). Therefore, if the effective spot size at $60^{\circ}$ incidence angle - which is the maximum spot size, as the lobe increases with $\cos \left(\theta_{\mathrm{i}}\right)$-must be kept to $18 \mathrm{~mm}(10 \%$ less than $20 \mathrm{~mm})$, then at $0^{\circ}$ incidence, this spot must have a maximum size of $9 \mathrm{~mm}$ (i.e. $18 \mathrm{~mm} / \cos \left(60^{\circ}\right)$ ). At $15^{\circ}$ incidence angle, the effective spot size must be a maximum of $9.318 \mathrm{~mm}$ (i.e. $9 \mathrm{~mm} \mathrm{x}$ $\left.\cos \left(15^{\circ}\right)\right)$, and so on. Figure 11 shows the mapped illumination spot for the VOSEG setup at $15^{\circ}$ incidence angle. The spot was mapped by illuminating the sample whilst the detector FOV was constrained to $1 \pm 0.1 \mathrm{~mm}$ using a pinhole baffle. The detector was then moved along the $\mathrm{x}$ - and $\mathrm{y}$ - planes $0.5 \pm 0.1 \mathrm{~mm}$ at a time. The spot has an effective size of $9 \pm 0.2 \mathrm{~mm}$ and therefore, the spot size at $60^{\circ}$ incidence angle will be $17.4 \pm 0.4 \mathrm{~mm}$ (within the desired range). (NB: It is not possible to map the spot at $0^{\circ}$ incidence, as the light source obstructs the detector.) 


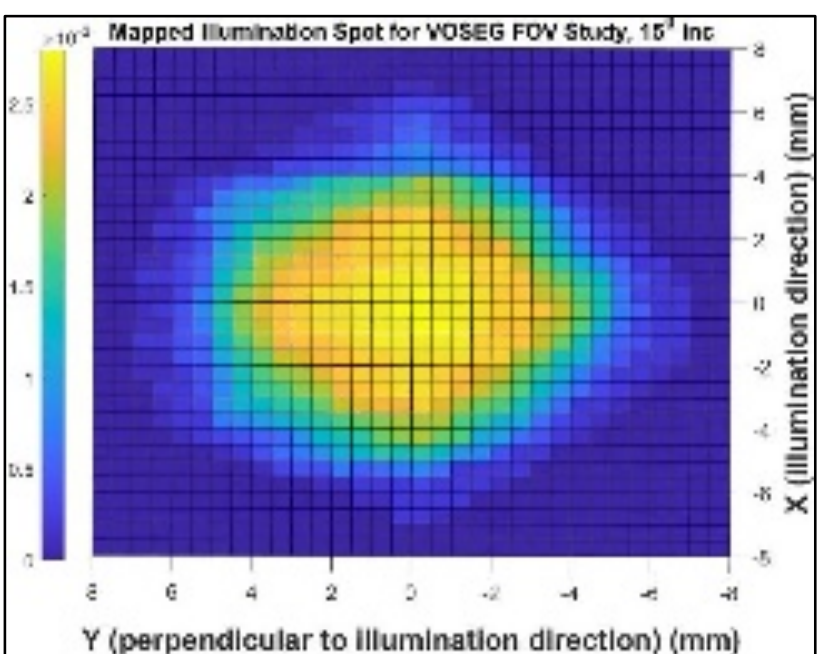

Figure 11: The illumination spot at $15^{\circ}$ incidence angle mapped for the Visible-Oxford Space Environment Goniometer. The effective spot size is $9 \pm 0.2 \mathrm{~mm}$. Z-axis is measured voltage in $V$.

Restricting the FOV to a $20 \mathrm{~mm}$ circle reduces the contribution from stray light in the lock-in output signal to the desirable range of $<2 \%$ of the measured BRDF, as is demonstrated by the agreement between White Spectralon measurements made by BUG and VOSEG for $15^{\circ}, 30^{\circ}, 45^{\circ}$ and $60^{\circ}$ incidence angles (see Part 3). However, the effective spot size must be as large as possible, without exceeding the maximum desired value of $18 \mathrm{~mm}$, to sample as much of the target surface as possible (and therefore to achieve maximum signal). If the effective illumination spot is any larger than $18 \mathrm{~mm}$, then variations in BRDF due to stray light may lead to systematic errors of $>2 \%$ which is larger than the average uncertainty due to the measured drift in the laser output ${ }^{45}$.

\section{Calibration Target Height and Tilt Sensitivity}

Two factors which may affect the alignment of the illuminated spot with regards to the FOV are the height and tilt of the White Spectralon calibration target (see Part 3$)^{46}$. For each experimental run, the height and tilt of the White Spectralon target are set using calipers and a spirit level, respectively. However, the human error in this method can lead to variations of height and tilt of up to $0.5 \mathrm{~mm}$ and $1^{\circ}$, respectively. The sensitivity of the White Spectralon BRDF measurements to such height and tilt changes is considered below.

\section{i. Height Sensitivity Study}

For the height sensitivity study, the White Spectralon target was first levelled using a spirit level — and set to $24 \pm 0.5 \mathrm{~mm}$ above the base plate of the VOSEG. BRDFs were then measured at $60^{\circ}$ incidence angle and over $0^{\circ}$ to $65^{\circ}$ reflectance angles, for five heights$24 \pm 0.5 \mathrm{~mm}, 25 \pm 0.5 \mathrm{~mm}, 26 \pm 0.5 \mathrm{~mm}$ (optimum), $27 \pm 0.5 \mathrm{~mm}$ and $28 \pm 0.5 \mathrm{~mm}$ above the base plate-in $1 \mathrm{~mm}$ steps. The optimal height was determined as $26 \pm 0.5 \mathrm{~mm}$ before this study by observing the output of the lock-in amplifier and noting the height at which the signal was at a maximum. This was used as the centre height of the study. Figure 12 shows BRDF measurements of White Spectralon made by VOSEG for the five heights in this study. It also shows BRDF measurements made by the Bloomsburg University Goniometer (BUG) of White Spectralon, which are the measurements VOSEG must match (to within one standard error), to ensure it is calibrated.

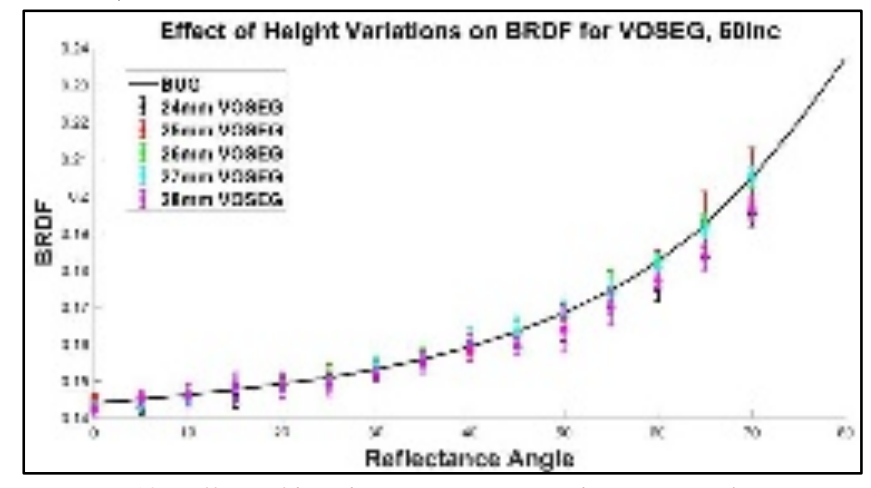

Figure 12: Effect of height variations on White Spectralon $B R D F$ measurements for VOSEG, at $60^{\circ}$ incidence angle, for five heights: corresponding to surfaces at $24 \pm 0.5,25 \pm 0.5,26 \pm 0.5$, $27 \pm 0.5$ and $28 \pm 0.5 \mathrm{~mm}$ above the base plate; and comparable Bloomsburg University Goniometer (BUG) measurements of White Spectralon.

Figure 12 shows that variations in the height of the White Spectralon calibration target of $<1 \mathrm{~mm}$ do not introduce variations in BRDF which are larger than the standard error in the measurements. Therefore, the height variation must not vary by $>1 \mathrm{~mm}$ over one experimental run (for the calibration target). Height variations larger than this lead to the illuminated spot drifting from within the centre of the FOV, which, for $60^{\circ}$ incidence angle 
will lead to systematic errors in the measurements of White Spectralon.

\section{ii. $\quad$ Tilt Sensitivity Study}

White Spectralon BRDFs were measured for a level surface, then for forwards-, backwards-, left- and right-tilted surfaces. To set the tilt, a spirit level was used. First, the surface was levelled, and then screws were tightened or loosened on the stage which holds the White Spectralon target, to adjust the tilt until the spirit level bubble was approximately half-way between the centre and the edge (in whichever direction was required). This resulted in angular variations of $2.00 \pm 0.05^{\circ}$, as shown in Figure 13:

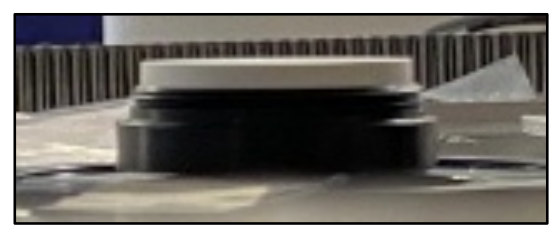

Figure 13: White Spectralon tilted backwards by $2 \pm^{0}$.

The height was set to $26 \pm 0.5 \mathrm{~mm}$ above the goniometer surface, as determined in Part $\underline{i}$. The results of the tilt study are shown, alongside the BUG BRDF measurements for White Spectralon, in Figure 14:

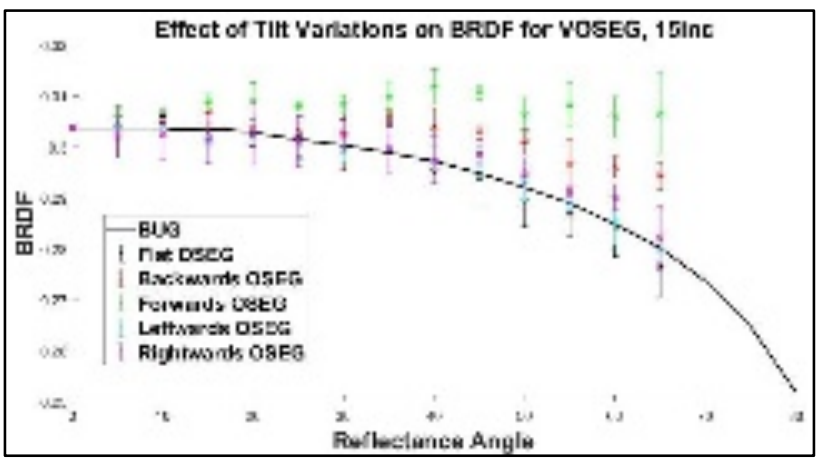

Figure 14: Effect of tilt variations on White Spectralon BRDF measurements for VOSEG, at $15^{\circ}$ incidence angle, for five tilts: corresponding to flat, backwards-tilting, forwards-titling, leftwards-tilting and rightwards-tilting surfaces; and comparable Bloomsburg University Goniometer (BUG) measurements of White Spectralon. $N B$ : this figure shows BRDFs with no angular postprocessing, i.e. assuming reflectance angles $0 / 5 / 10 \ldots 65^{\circ}$. (Normalised at $0^{\circ}$ reflectance angle to BUG.)

To show how post-processing can resolve this issue - as done with measurements taken by the PHIRE-1 and PHIRE-2 goniometer systems ${ }^{23}-2^{\circ}$ reflectance angle changes can be incorporated into the BRDF calculations (i.e. using $\cos \left(2 / 7 / 12 \ldots 67^{\circ}\right)$ instead of $\cos \left(0 / 5 / 10 \ldots 65^{\circ}\right)$ to calculate the angular dropoff of the curve). The corrected BRDFs of the flat, and tilted BRDF measurements are shown in Figure 15, and all agree with BUG within one standard error:

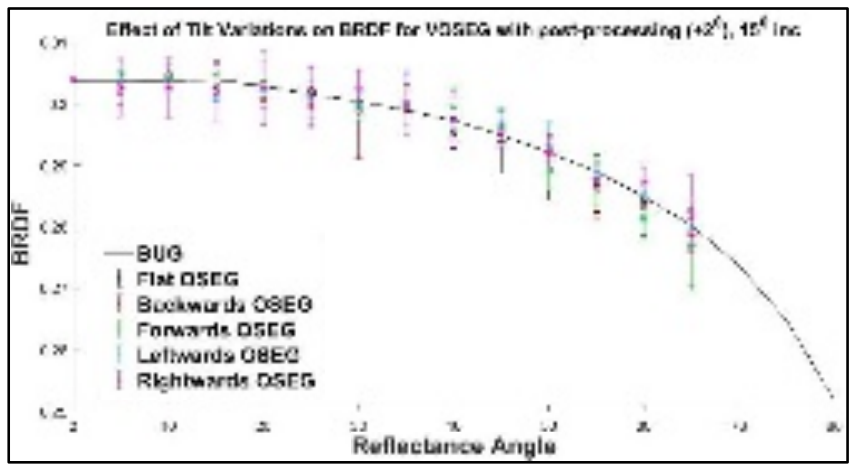

Figure 15: Effect of tilt variations on White Spectralon $B R D F$ measurements for VOSEG, at $15^{\circ}$ incidence angle, for five tilts: corresponding to flat, backwards-tilting, forwards-titling, leftwards-tilting and rightwards-tilting surfaces; and comparable Bloomsburg University Goniometer (BUG) measurements of White Spectralon. $N B$ : this figure shows BRDFs with angular postprocessing: i.e. $2^{\circ}$ angular variations are used within the BRDF calculation, using cosine $\left(2 / 7 / 12 \ldots 67^{\circ}\right)$ as opposed to cosine $\left(0 / 5 / 10 \ldots 65^{\circ}\right)$. (Normalised at $0^{\circ}$ reflectance angle to $B U G$.)

It is clear that for well-aligned BRDF measurements to be taken, sample surfaces must be at a height of $26 \pm 0.5 \mathrm{~mm}$ above the base plate, with variations no larger than $1 \mathrm{~mm}$; and must not have any significant $\left(>1^{\circ}\right)$ forwards- or backwards-tilt to them relative to the measurement plane (i.e. the sample stages must be levelled using a spirit level before each run), or else post-processing must remove the effect on BRDFs due to tilting. Any more than $1 \mathrm{~mm}$ height variations, or $1^{\circ}$ tilt variations, leads to BRDFs which are not in agreement with BUG measurements, within one standard error.

VOSEG's requirements can therefore be updated to include: tilt control of $<0.5^{\circ}$ for each experimental run, or else post-processing must be done; height-control of $<1 \mathrm{~mm}$ over each experimental run to ensure BRDF variations of $<2 \%$ (which is within the average uncertainty due to the laser's output instability); and locational variations of the illuminated spot which are $<1 \mathrm{~mm}$, to ensure 
the spot is within the FOV for all incidence angle measurements.

\section{Validation by Comparing White Spectralon Measurements to the Bloomsburg University Goniometer}

White Spectralon is a well-characterised commercial fluoropolymer material, which can used as a reflectance reference target at visible wavelengths ${ }^{46}$. BUG measured White Spectralon for Foote et al.'s 2010 study as a reference target before taking BRDF measurements of Apollo 11 (10084) and 16 (68810) regolith samples ${ }^{11}$. VOSEG is also calibrated by taking reflectance measurements of White Spectralon ${ }^{46}$. With VOSEG, BRDF measurements of samples are calculated from measured lock-in amplifier voltages using the following method:

- Lock-in amplifier voltages for Spectralon, $V_{\text {Spec }}$, are measured as a function of viewing angles (i.e. over reflectance angle, as in Figures 16, 17, 18 and 19).

- Lock-in amplifier voltages for the sample, $V_{\text {Sample }}$ are measured as a function of reflectance angle.

- Using the known reflectance values of White Spectralon ${ }^{48}, r_{\text {spec }}$, at the appropriate viewing angles, the BRDF of the sample can be calculated-from the reflectance of the sample, $r_{\text {Sample }}$ - using Equations 8 and 9 (NB: Dividing by cosine (inc) in Equation 8 is necessary due to the design choice of having an illumination spot which is smaller than the field of view. This is because of the difficulty in creating an illumination spot which is spatially uniform.):

$$
\operatorname{BRDF}_{\text {Sample }}\left(\theta_{r}\right)=\frac{r_{\text {Sample }}\left(\theta_{r}\right)}{\cos (i)}
$$

Where,

$$
r_{\text {Sample }}\left(\theta_{r}\right)=\frac{r_{\text {Spec }}\left(\theta_{r}\right)}{V_{\text {Spec }}\left(\theta_{r}\right)} \times V_{\text {Sample }}\left(\theta_{r}\right)
$$

Figures 16, 17, 18 and 19 show BRDF measurements taken by VOSEG and BUG for White Spectralon at $15^{\circ}, 30^{\circ}, 45^{\circ}$ and $60^{\circ}$ incidence angles, respectively, in the Principle Plane ( $180^{\circ}$ azimuthal angle).

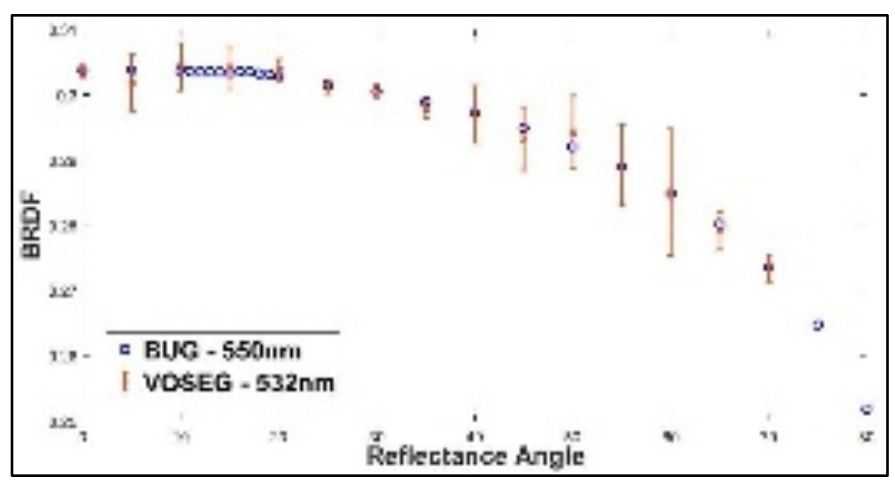

Figure 16: Bidirectional Distribution Function measurements of White Spectralon, comparing the Bloomsburg University Goniometer to the Oxford Space Environment Goniometer for $15^{\circ}$ incidence angle, in the Principle Plane (180 azimuthal).

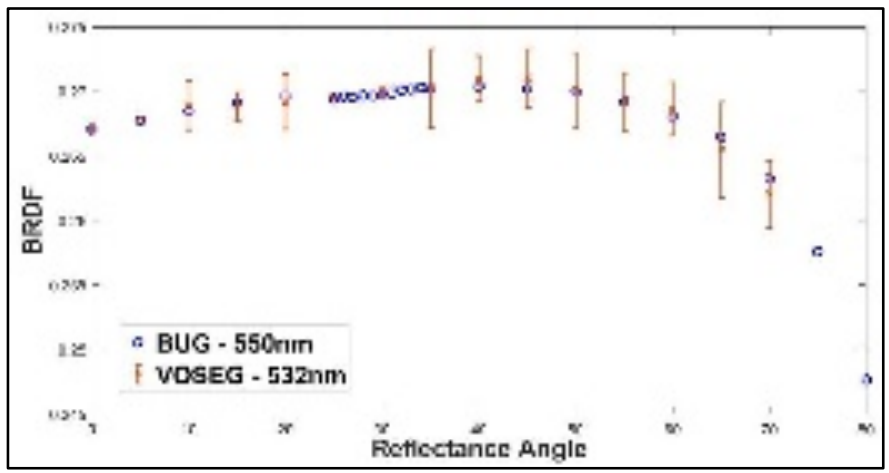

Figure 17: Bidirectional Distribution Function measurements of White Spectralon, comparing the Bloomsburg University Goniometer to the Oxford Space Environment Goniometer for $30^{\circ}$ incidence angle, in the Principle Plane (180 azimuthal).

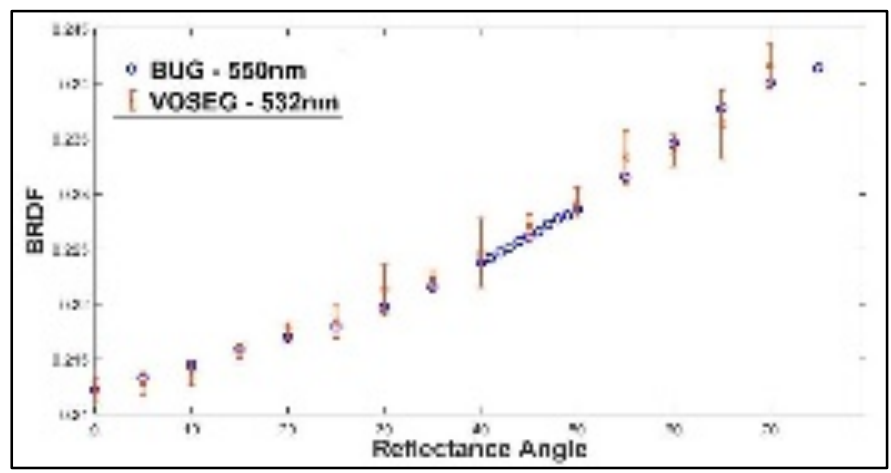

Figure 18: Bidirectional Distribution Function measurements of White Spectralon, comparing the Bloomsburg University Goniometer to the Oxford Space Environment Goniometer for $45^{\circ}$ incidence angle, in the Principle Plane (180 azimuthal).

VOSEG's BRDF measurements of Spectralon are in agreement with those taken by BUG, within standard error (calculated as the standard deviation over three measurements). 


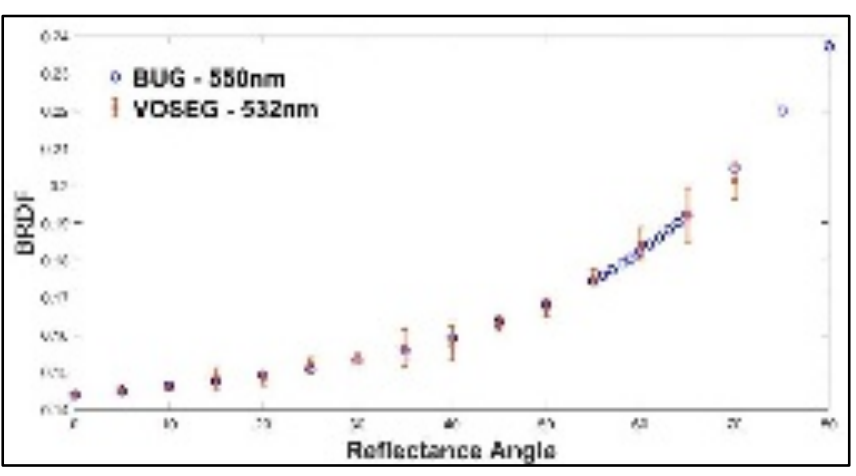

Figure 19: Bidirectional Distribution Function measurements of White Spectralon, comparing the Bloomsburg University Goniometer to the Oxford Space Environment Goniometer for $60^{\circ}$ incidence angle, in the Principle Plane (180 azimuthal).

\section{Conclusions}

The OSEG setup has been modified to measure visible wavelength BRDFs in ambient conditions over a range of viewing angles (0$60^{\circ}$ incidence, $0-70^{\circ}$ reflectance, $\sim 4-130^{\circ}$ phase, $0-360^{\circ}$ azimuthal). White Spectralon measurements taken by VOSEG agree (within the standard error) with those taken by BUGthe goniometer system which was used to measure Apollo regolith samples in the Foote et al. 2010 study $^{11}$. VOSEG can measure down to $\sim 4^{\circ}$ phase angles-which means it can measure part of the opposition effect-and it can take measurements over a range of angles, up to and including $60^{\circ}$ incidence and $70^{\circ}$ reflectance angles.

The illumination spot of the light source has been mapped. The FOV has also been mapped and has an effective diameter of $\sim 20 \mathrm{~mm}$. Height and tilt sensitivity studies for the calibration target have been performed, and these show that to be able to make measurements of White Spectralon which are in agreement with BUG, the height must remain $26 \pm 1 \mathrm{~mm}$ above the base plate of the goniometer, and the tilt of the sample must not be greater than $1^{\circ}$, or else post-processing must be done to correct for this.

This goniometer system has been updated and characterised, so it is able to take ground support measurements, now and in future, for missions such as the Diviner Lunar

Radiometer ${ }^{35}$, Hayabusa-2 (Asteroid -162173 Ryugu $^{48}$, BepiColombo (Mercury) ${ }^{49}$, OSIRISRex (Asteroid - Bennu) ${ }^{50}$ and other planned future missions such as Lunar Trailblazer and Comet Interceptor ${ }^{51}$.

\section{Acknowledgements}

The authors would like to acknowledge the financial support of the Science and Technology Facilities Council (STFC), the Royal Astronomical Society (RAS) and Trinity College, Oxford. They would also like to thank Jon Temple, Andy Clack and Jordan Hoe for their support in the laboratory workshop. Finally, they would like to thank Professor Michael Shepard, Professor David Paige and Dr Emily Foote for their correspondence and guidance in advance of this work.

\section{Data Availability}

The data that support the findings of this study are available from the corresponding author upon reasonable request.

\section{References}

1. Hapke, B. J. Geophys. Res. Solid

Earth, (1981).

2. Hapke, B. Icarus, (2012).

3. Shepard, M. K. \& Helfenstein, P. J. Geophys. Res. E Planet, 112, 1-17 (2007).

4. Hudson, R. S. \& Ostro, S. J. Icarus, (1999).

5. Simonelli, D. P., Veverka, J., Thomas, P., Helfenstein, P., Carcich, B. \& Belton, M. Icarus, (1996).

6. Sato, H., Robinson, M. S., Hapke, B., Denevi, B. W. \& Boyd, A. K. J. 
Geophys. Res. E Planets, (2014).

7. Chin, G. \& Team, Diviner. Space Sci. Rev., (2007).

8. McEwen, A. S. Icarus, (1991).

9. Helfenstein, P. \& Shepard, M. K. Icarus 141, 107-131 (1999).

10. Hapke, B. Icarus, (1984).

11. Foote, E., AGU Fall Meet. Abstr. 1499, (2010).

12. Morris, R. V. Handbook of Lunar Soils: Part I: Apollo 11-15, (1983).

13. O. King, T. Warren, N. E. Bowles, E. Sefton-Nash, R. Fisackerly, R. T. Planet. Space Sci., (2019).

14. Vasavada, A. R., Bandfield, J. L., Greenhagen, B. T., Hayne, P. O., Siegler, M. A., Williams, J. P., Paige, D. A. J. Geophys. Res. E Planets 117, (2012).

15. Hayne, P. O., Hendrix, A., SeftonNash, E., Siegler, M. A., Lucey, P. G., Retherford, K. D., Williams, J. P., Greenhagen, B. T., Paige, D. A. Icarus, (2015).
16. Paige, D. A. \& Team, Diviner, Science (80-. ), (2010).

17. Warren, T., Doctoral Thesis, (2015).

18. Hapke, B. Cambridge University Press, (2011).

19. McGuire, A. F. \& Hapke, B. W. Icarus, (1995).

20. Cruikshank, D. P., Roush, T. L., Geballe, T. R., Owen, T. C., de Bergh, Cash, M. D., Hartmann, W. K. Icarus, (2001).

21. Poulet, F., Cuzzi, J. N., Cruikshank, D. P., Roush, T. \& Dalle Ore, C. M. Icarus, (2002).

22. Shepard, M. K. \& Helfenstein, P. J. Geophys. Res. E Planets, (2007).

23. Gunderson, K., Thomas, N. \& Whitby, J. A. Planet. Space Sci., (2006).

24. Yoldi, Z., Pommerol, A., Jost, B., Poch, O., Gouman, J., Thomas, N. Geophys. Res. Lett., (2015).

25. Potin, S. M., Brissaud, O., Beck, P., Schmitt, B., Magnard, Y., Correia, J. J., Rabou, P., Jocou, L. Appl. Opt., (2018). 
26. Biggar, S. F., Thome, K. J. \&

Wisniewski, W. IEEE Trans. Geosci.

Remote Sens., (2003).

27. Johnson, J., Lunar Planet., (2008).

28. Squyres, S. W. \& Veverka, J. Icarus

50, 115-122 (1982).

29. Hapke, B. Icarus, (2002).

30. Hendrix, J. \& Carman, C. H.

Renaissance Theories of Vision

Introduction. Renaissance Theories of

Vision, (2010).

31. Shepard, M. K. \& Helfenstein, P. Icarus, (2011).

32. Hapke, B. Theory reflectance Emit. Spectrosc., (1993).

33. Bandfield, J. L., Hayne, P. O., Williams, J. P., Greenhagen, B. T. \& Paige, D. A. Icarus 248, 357-372 (2015).

34. Rozitis, B. \& Green, S. F., Mon. Not. R. Astron. Soc., (2011).

35. Paige, D. A. \& Team, Diviner., Space Sci. Rev., (2010).

36. Lazbin, I. K. I., Rassas, M. M. M. \&
Shamordola, L. S. K. Instrument. Space Sci Rev, (2018).

37. Hayne, P. O. \& Team, Diviner., $J$. Geophys. Res. Planets, (2017).

38. Mitchell, J. K., Carrier III, W. D., Costes, N. C., Houston, W. N., \& Scott, R. F. Lunar Planet. Sci. Conf. 260, (1973).

39. Woods-Robinson, R., Siegler, M. A. \& Paige, D. A. J. Geophys. Res. Planets, (2019).

40. McEwen, A. S. U.S. Geol. Surv., (1996).

41. Vasavada, A. R., Paige, D. A. \& Wood, S. E. Icarus, (1999).

42. Whipple, A. L. Adv. Sp. Res., (1993).

43. Warren, T. J., Bowles, N. E., Donaldson Hanna, K. \& Thomas, I. R. Rev. Sci. Instrum., (2017).

44. Nelson, R. M., Smythe, W. D., Hapke, B. W. \& Hale, A. S. Planet. Space Sci., (2002).

45. ThorLabs. CPS532 Specification Sheet, 5-6, (2013) 
46. Labsphere. Spectralon ${ }^{\circledR}$ Diffuse

Reflectance Standards: Datasheet, 1-4, (2017).

47. Bentham. Bentham ILI QTH Source:

Datasheet,1-2, (2017).

48. Kawaguchi, J., Fujiwara, A. \& Uesugi,

T. Acta Astronaut., (2008).

49. Benkhoff, J. \& Team, BepiColombo.,

Planet. Space Sci., (2010).

50. Lauretta, D. \& Team, O.-REx., Lunar

Planet. Inst. Sci., (2012).

51. Snodgrass, C. \& Jones, G. H. Nature

Communications, (2019). 


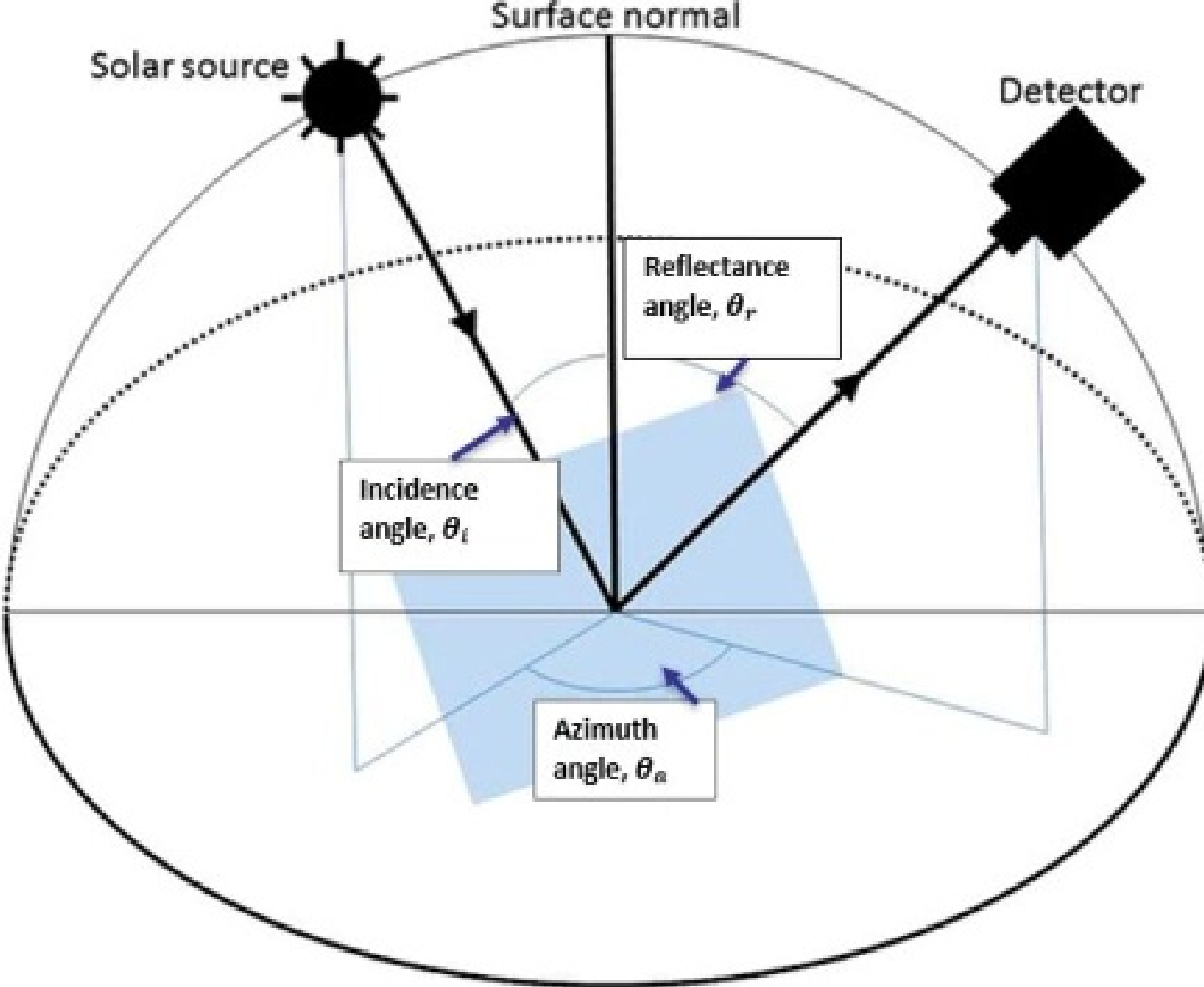




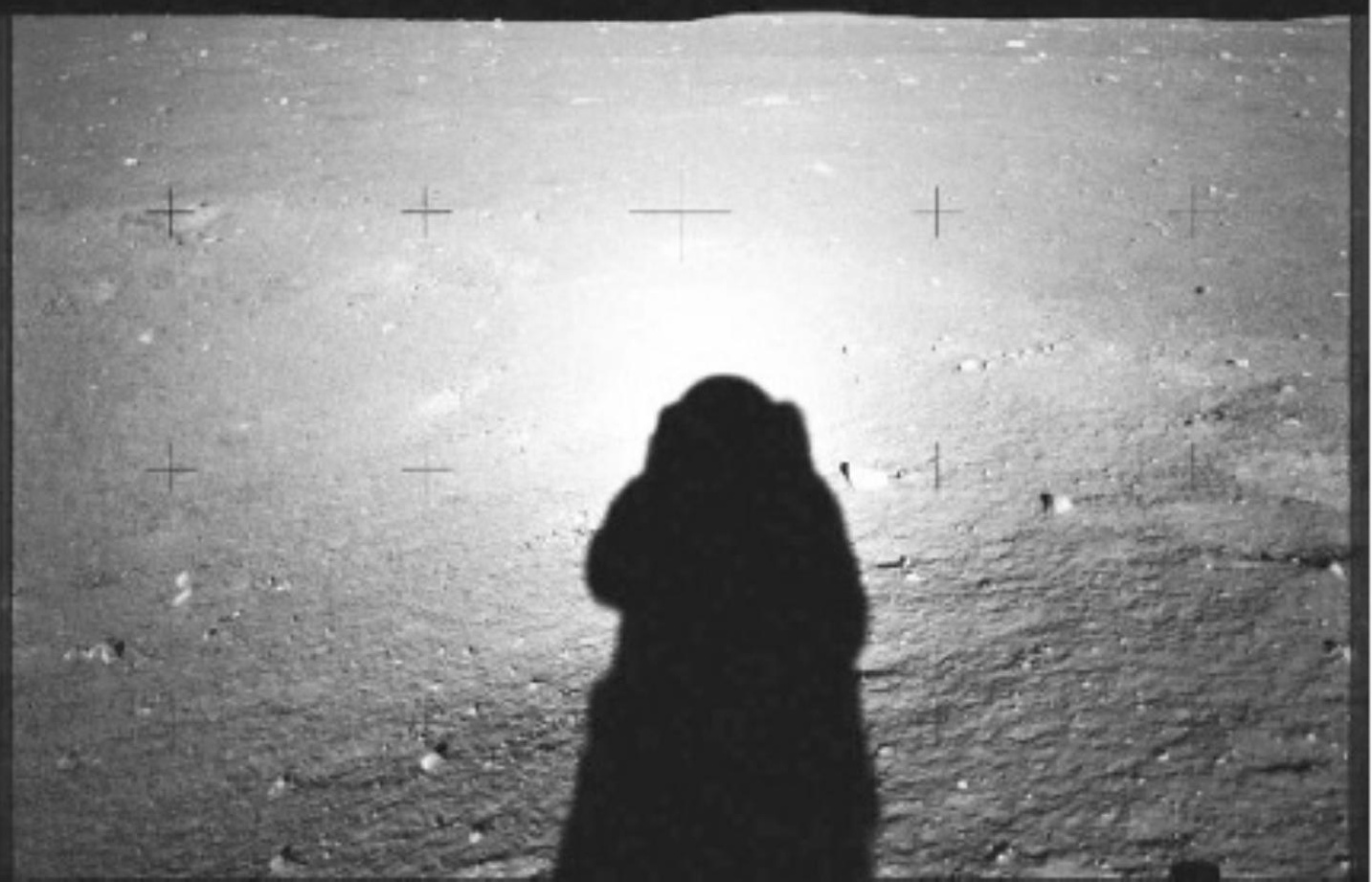




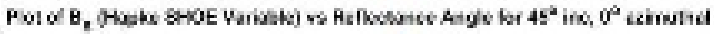

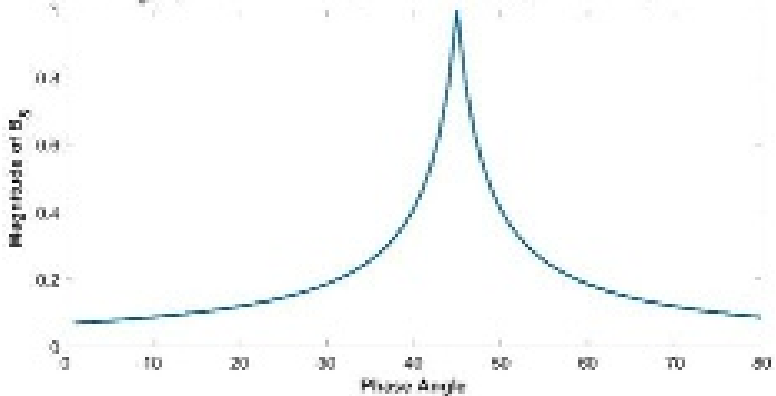




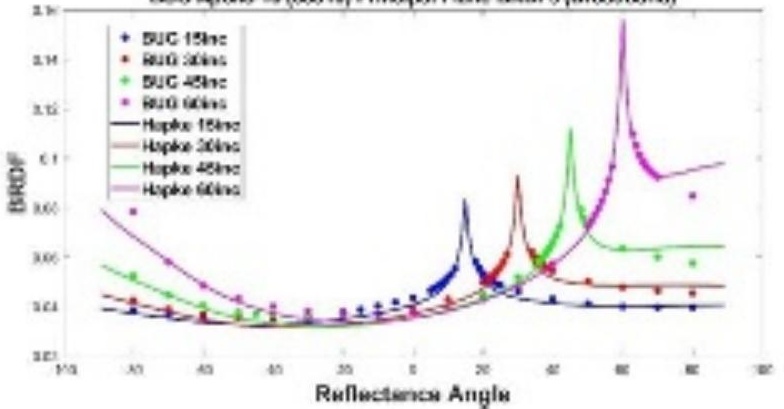




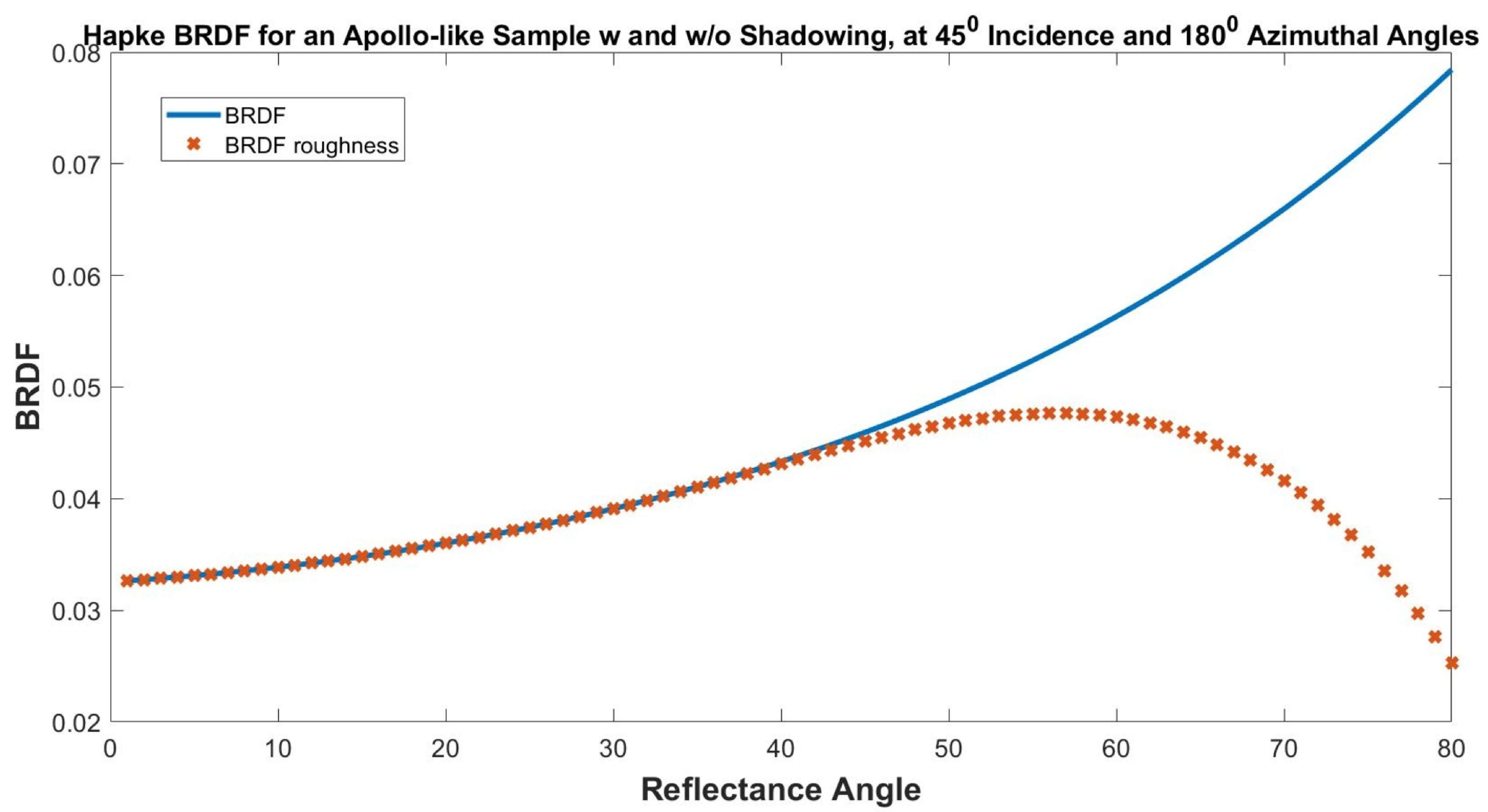




\section{Re-radiation}

\section{Permanent illumination}

Permanent shaded region (PSR) 


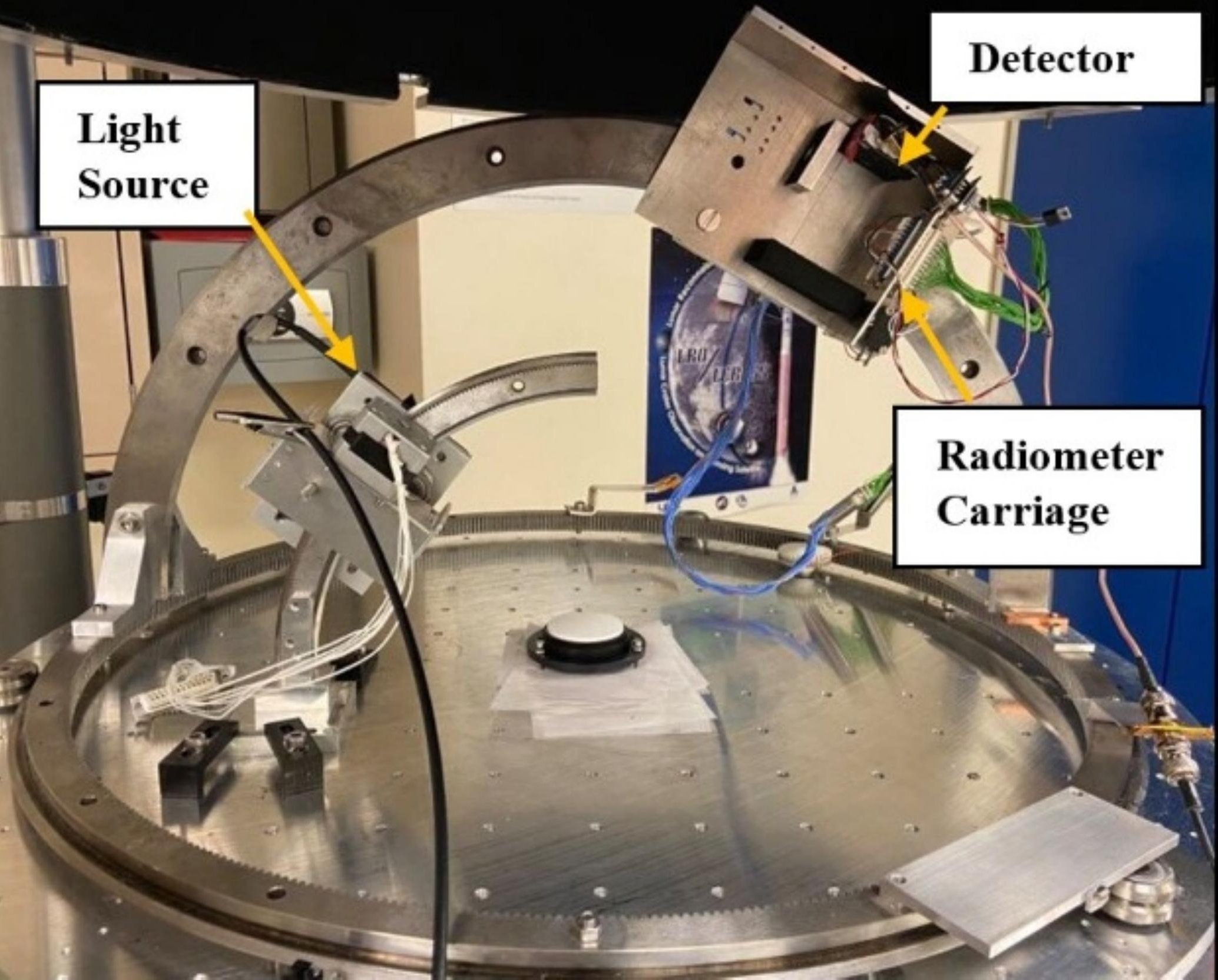




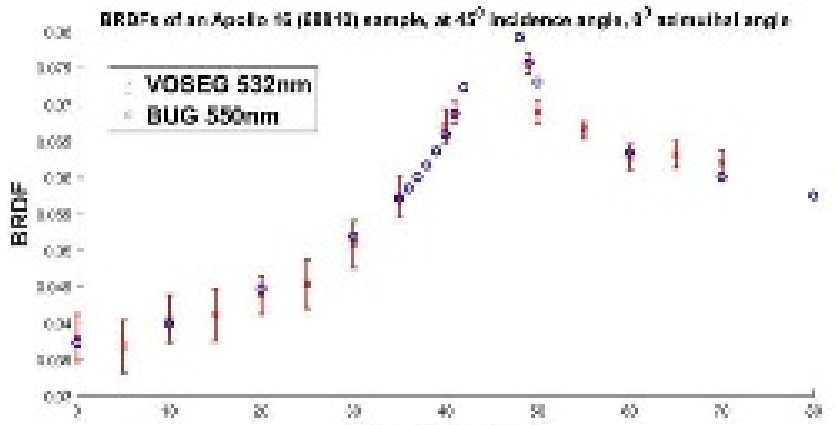

Reflectance Angle 
Low incidence angle Light source holder 


\section{vOSEG Messured Field of View [FON]}

E

s

$+s$

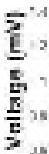

15

$94-$

Xs

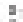

$x \times$

ects

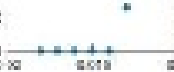

$a+c+a+a+b$

+ Maasurad FOW

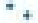

4

tet

2.000

8

soce

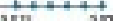

Dixtance $(\mathrm{m})$ 
$\times 10^{3}$ Xapfed Illuminalian Spot for VOSEO FOV Stucly, $15^{0} \mathrm{kn}$

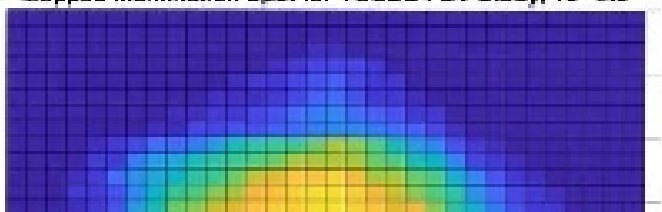


Effoct of Helght Varlations on BRDF for VOSEG. 60inc

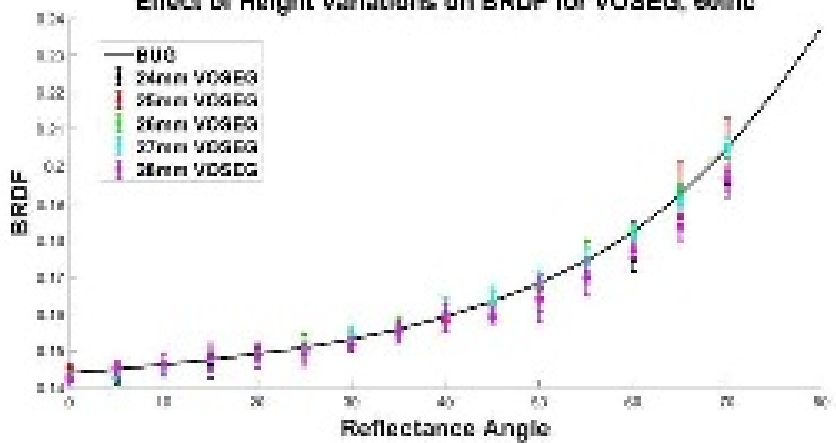


a Funuy Ploce .

merol of space exuleration seen athe eres ef cartoonists

,

6.

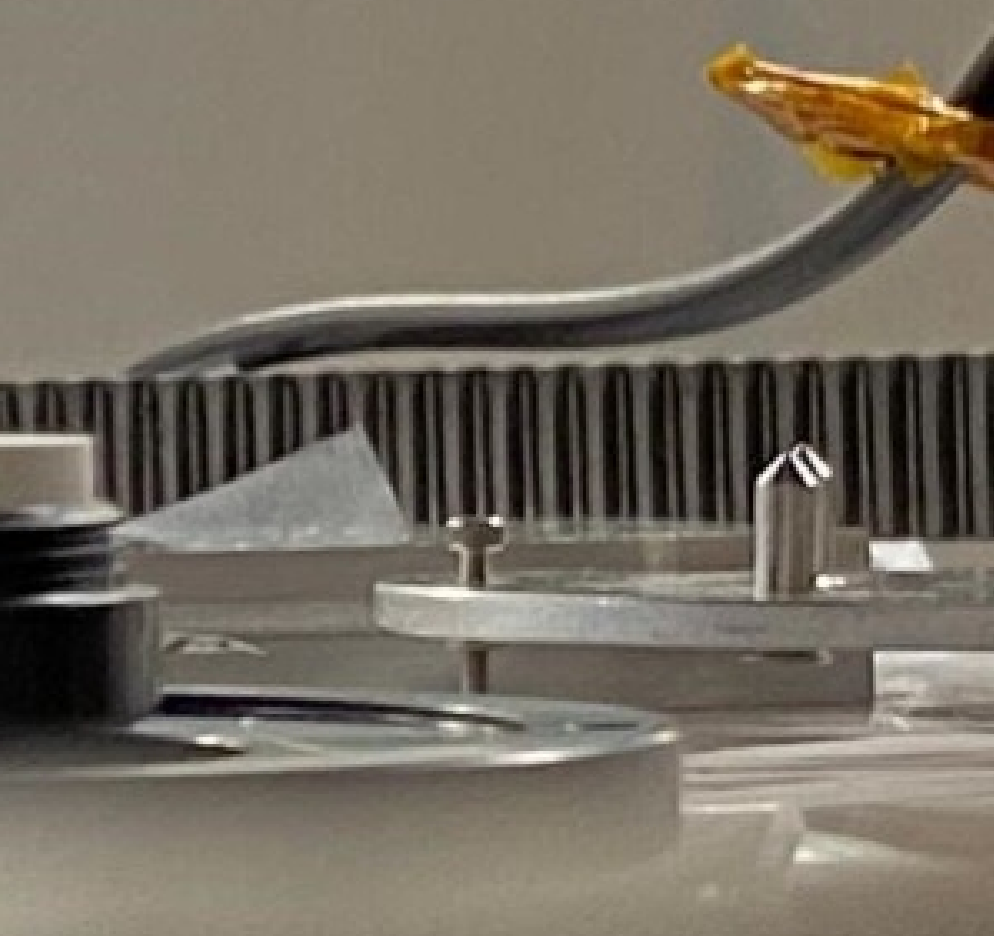




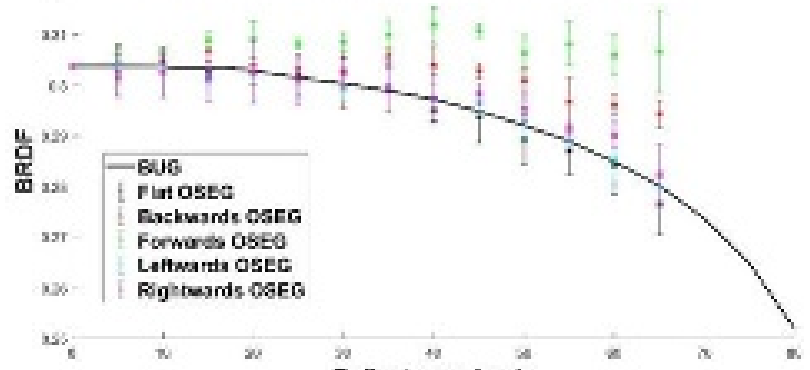

Reflectances Angle 
0.31 Effect of Tilt Variations on BRDF for VOSEG with post-processing $\left(+2^{0}\right), 15^{0}$ inc

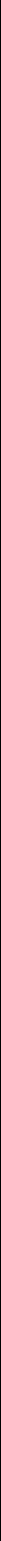




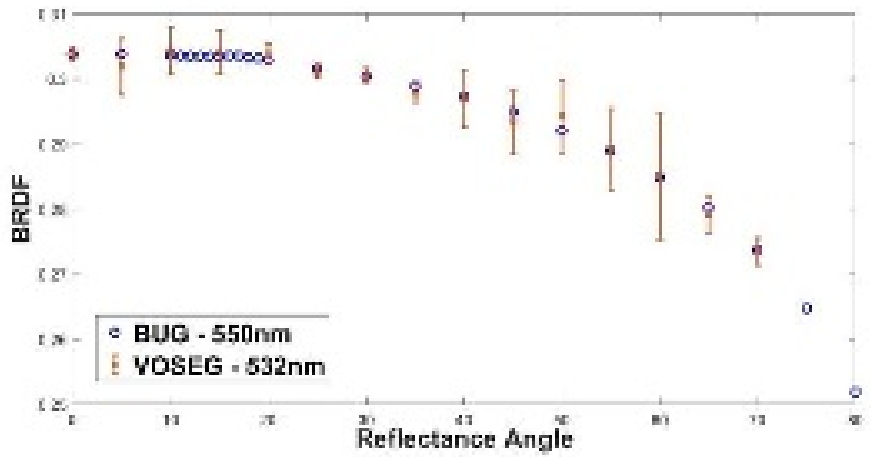




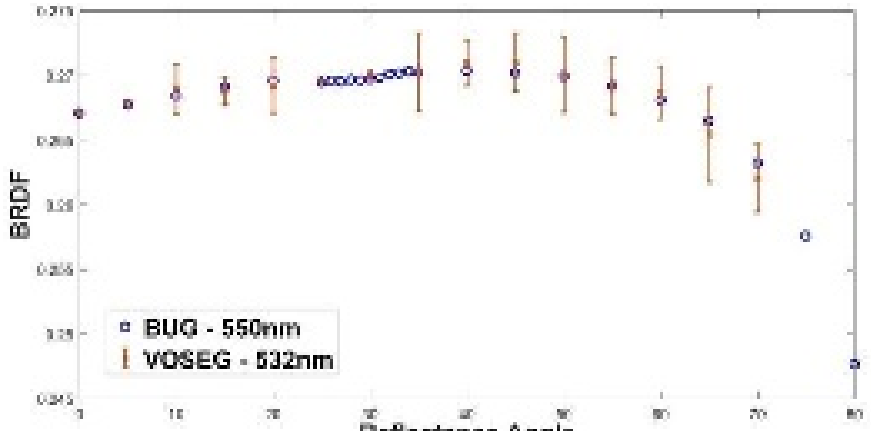

Reflectance Angle 


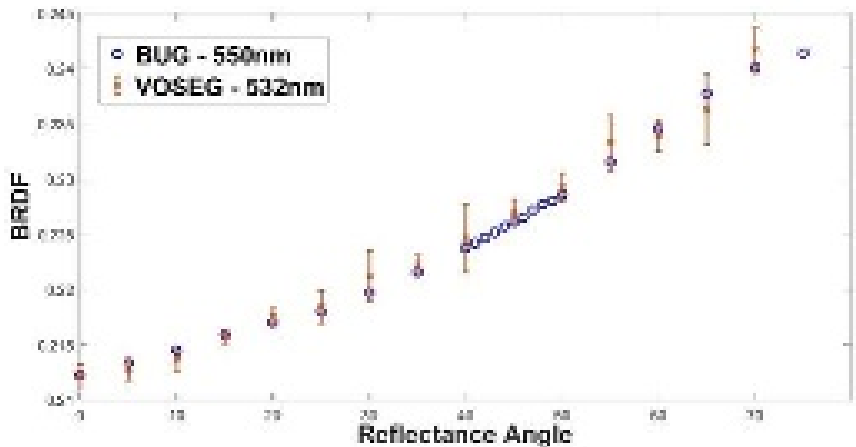


sid

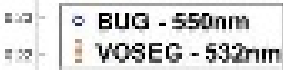

$8 x$

$\frac{\frac{\rho}{\alpha}}{\frac{0}{\alpha}}=1$ III

217

816

is $=14$

w

I

8

a)

n

Reflectanoe Angle 